Chirurg 2020 $91: 891$

https://doi.org/10.1007/s00104-020-01282-9

Online publiziert: 15 . September 2020

(c) Springer Medizin Verlag GmbH, ein Teil von Springer Nature 2020

\section{Originalpublikation}

Mazzaferro V, Citterio D, Bhoori S et al (2020) Liver transplantation in hepatocellular carcinoma after tumour downstaging (XXL): a randomised, controlled, phase $2 \mathrm{~b} / 3$ trial. Lancet Oncol 21: 947-956

Einleitung. Durch Lebertransplantation bei Patienten mit hepatozellulärem Karzinom (HCC) innerhalb der $\mathrm{Mi}$ lan-Kriterien können hervorragende Langzeitergebnisse mit 5-Jahres-Überlebensraten (5 JÜR) von über $75 \%$ erreicht werden. Lokoregionale Verfahren können die morphologische Tumorlast soweit „downstagen“, dass die MilanKriterien wieder erfüllt werden. Ziel der vorliegenden Studie war es, das Outcome bei Patienten nach erfolgreichem Downstaging bei HCC und Lebertransplantation mit Fortführung lokoregionaler/systemischer Therapieverfahren in Bezug auf Rezidivraten sowie Langzeitüberleben zu vergleichen.

Methodik. In dieser italienischen, multizentrischen, randomisiert kontrollierten Studie (RCT) wurden zwischen 2011 und 201545 Patienten mit HCC außerhalb der Milan-Kriterien, ohne extrahepatische Tumorlast oder Gefäßinvasion, im Alter zwischen 18 und 66 Jahren nach erfolgreichem Tumordownstaging (transarterielle Chemoembolisation, selektive interne Radiotherapie, Radiofrequenzablation und Resektion in Kombination oder alleine) 3 Monate nach Abschluss der initialen Therapie randomisiert und 23 Patienten (51\%) der Transplantationsgruppe und 22 Patienten (49\%) der Kontrollgruppe zugeteilt. Patienten der

Johannes Fritsch • Michael Ardelt ${ }^{1}$ - Utz Settmacher

'Klinik für Allgemein-, Viszeral- und Gefäßchirurgie, Jena, Deutschland

\title{
Lebertransplantation nach Tumordownstaging bei hepatozellulärem Karzinom
}

Kontrollgruppe erhielten beim Nachweis eines Tumorprogresses erneute lokoregionale und/oder systemische Therapieverfahren. Primäre Endpunkte waren die Rate des tumorfreien/progressionsfreien Überlebens nach 5 Jahren sowie die 5 JÜR. Sekundär wurden Komplikationen innerhalb der jeweiligen Gruppen untersucht.

Ergebnisse. Die mediane Dauer des Downstagings betrug 6 Monate (IQR 4-11 Monate) bei einer Responderrate von $73 \%$. Die mediane Follow-up-Zeit betrug 71 Monate (IQR 60-85 Monate). In der Transplantationsgruppe lagen zwischen Beginn des Downstagings und der Transplantation 12 Monate im Median, zwischen Randomisierung und Transplantation 3 Monate. Während der Nachbeobachtungszeit verstarben 5 Patienten der Transplantationsgruppe (23\%) und 16 Patienten der Kontrollgruppe (73\%), dies entspricht einem 5 JÜR von 77,5\% (95\%-KI 61,9-97,1) in der Transplantationsgruppe vs. $31,2 \%$ (95\%-KI $16,6-58,5)$ in der Kontrollgruppe (HR 0,32; $95 \%$-KI 0,11-0,92; p:0,035). Die Rate des tumorfreien/progressionsfreien Überlebens nach 5 Jahren betrug in der Transplantationsgruppe $\quad 76,8 \% \quad(95 \%$ KI 60,8-96,9), in der Kontrollgruppe lag die Rate des progressionsfreien Überlebens innerhalb von 5 Jahren bei nur 18,3\% (95\%-KI 7,1-47,0; HR 0,2; $95 \%$-KI 0,07-0,57; p:0,003), wobei der erneute Tumorprogress bei $56 \%$ der Patienten bereits innerhalb der ersten 12 Monate nach Therapieende auftrat. Die häufigsten schwerwiegenden Komplikationen waren Hepatitis-C-Rezidive (3 Patienten, $23 \%$ ) und akute Transplan- tatabstoßung (2 Patienten, 9\%) in der Transplantationsgruppe sowie Postembolisationssyndrome (2 Patienten, 9\%) in der Kontrollgruppe.

\section{Kommentar}

Aufgrund veränderter Bestimmungen bezüglich Spenderauswahl und Dringlichkeitsbeurteilung zur Transplantation bei HCC in Italien wurde die Studie vorzeitig abgebrochen, weshalb die randomisierte Patientenzahl mit 45 gering und die Aussagekraft eingeschränkt ist. In vorangegangenen, nichtrandomisierten Studien konnten bereits gute Langzeitergebnisse nach Transplantation bei erfolgreichem Downstaging gezeigt werden. Die Ergebnisse der vorliegenden RCT bestätigen dies eindrücklich mit signifikant erhöhten Raten an Gesamtüberleben sowie tumorfreiem Überleben in der Transplantationsgruppe. Sicher eine weitere Studie, die das biologische Tumorverhalten als Prognosekriterium beschreibt und zukünftig bei der Listung von Patienten zur Lebertransplantation Berücksichtigung finden sollte.

\section{Korrespondenzadresse}

PD Dr. med. habil. Dr. med. univ. Michael Ardelt

Klinik für Allgemein-, Viszeral- und Gefäßchirurgie

Am Klinikum 1, 07747 Jena, Deutschland Michael.Ardelt@med.uni-jena.de

Interessenkonflikt. J. Fritsch, M. Ardelt und U. Settmachergeben an, dass kein Interessenkonflikt besteht. 